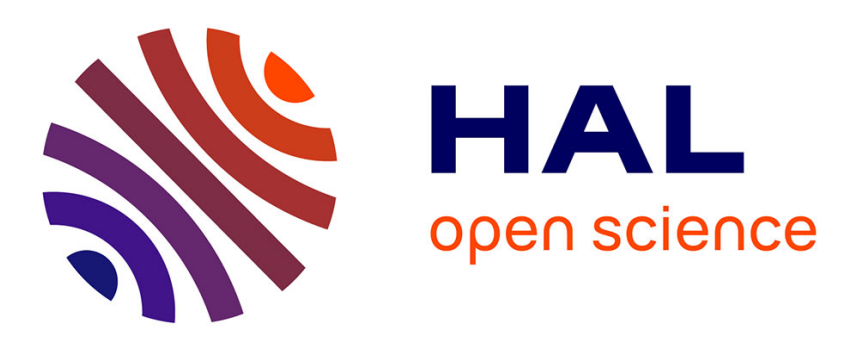

\title{
Influence of transport mechanisms on nucleation and grain structure formation in DC cast aluminium alloy ingots
}

\author{
M. Bedel, Miha Založnik, A. Kumar, H. Combeau, P. Jarry, E Waz
}

\section{To cite this version:}

M. Bedel, Miha Založnik, A. Kumar, H. Combeau, P. Jarry, et al.. Influence of transport mechanisms on nucleation and grain structure formation in DC cast aluminium alloy ingots. IOP Conference Series: Materials Science and Engineering, 2012, 27, 10.1088/1757-899X/27/1/012070 . hal-01710317

\section{HAL Id: hal-01710317 \\ https://hal.univ-lorraine.fr/hal-01710317}

Submitted on 15 Feb 2018

HAL is a multi-disciplinary open access archive for the deposit and dissemination of scientific research documents, whether they are published or not. The documents may come from teaching and research institutions in France or abroad, or from public or private research centers.
L'archive ouverte pluridisciplinaire HAL, est destinée au dépôt et à la diffusion de documents scientifiques de niveau recherche, publiés ou non, émanant des établissements d'enseignement et de recherche français ou étrangers, des laboratoires publics ou privés. 
Related content

\section{Influence of transport mechanisms on nucleation and grain structure formation in DC cast aluminium alloy ingots}

To cite this article: M Bedel et al 2012 IOP Conf. Ser.: Mater. Sci. Eng. 27012070

View the article online for updates and enhancements.
- Numerical study of the impact of inoculan and grain transport on macrosegregation and microstructure formation during solidification of an $\mathrm{Al}-22 \% \mathrm{Cu}$ alloy K O Tveito, M Bedel, M Založnik et al.
The effect of finite microscopic liquid solute diffusion on macrosegregation
Kormation
- Heterogeneous nucleation in
hypermonotectic aluminum allovs
M Köhler, L Ratke, I Kaban et al.

\section{Recent citations}

$\frac{\text { Process-scale modelling of microstructure }}{\text { in direct chill casting of aluminium alloys }}$ 


\title{
Influence of transport mechanisms on nucleation and grain structure formation in DC cast aluminium alloy ingots
}

\author{
M Bedel ${ }^{1}$, M Založnik ${ }^{1}$, A Kumar ${ }^{1}$, H Combeau $^{1}$, P Jarry $^{2}$ and E Waz $^{2}$ \\ ${ }^{1}$ Institut Jean Lamour, CNRS - Nancy-Université - UPV-Metz, \\ Ecole des Mines de Nancy, Parc de Saurupt CS 14234, F-54042 Nancy cedex, France \\ 2 Constellium Centre de Recherches Voreppe, \\ 725, rue Aristide Bergès, BP 27, F-38341 Voreppe cedex, France \\ E-mail: marie.bedel@ijl.nancy-universite.fr
}

\begin{abstract}
The grain structure formation in direct chill (DC) casting is directly linked to nucleation, which is generally promoted by inoculation. Inoculation prevents defects, but also modifies the physical properties by changing the microstructure. We studied the coupling of the nucleation on inoculant particles and the grain growth in the presence of melt flow induced by thermosolutal convection and of the transport of freefloating equiaxed grains. We used a volume-averaged two-phase multiscale model with a fully coupled description of phenomena on the grain scale (nucleation on grain refiner particles and grain growth) and on the product scale (macroscopic transport). The transport of inoculant particles is also modeled, which accounts for the inhomogeneous distribution of inoculant particles in the melt. The model was applied to an industrial sized ( $350 \mathrm{~mm}$ thick) DC cast aluminium alloy ingot. A discretised nuclei size distribution was defined and the impact of different macroscopic phenomena on the grain structure formation was studied: the zone and intensity of nucleation and the resulting grain size distribution. It is shown that nucleation in the presence of macroscopic transport cannot be explained only in terms of cooling rate, but variations of composition, nuclei density and grain density, all affected by transport, must be accounted for.
\end{abstract}

\section{Introduction}

In DC casting of aluminium alloys, inoculation is commonly used in the industry to refine the grain size and to homogenise the microstructure. Nadella and coworkers [1] showed this in controlled experiments on billets from two different alloys. They also observed that the grain size slightly increases from the centerline to the surface of the product. The grains nucleate on inoculant particles floating in the liquid melt, first as free-floating equiaxed grains, which at some point settle or coalesce into a packed structure, where the solidification then continues up to a fully solid structure. Depending on their motion through the solidification zone, the origin and the growth evolution of the grains can be multiple and duplex structures composed of coarse- and fine-cell dendrites can form, as shown by Nadella et al. [2]. The athermal heterogeneous nucleation on inoculant particles appears to be central in the formation of these microstructures. Inoculation is directly linked to the maximum undercooling reached: the higher the undercooling, the more inoculant particles are activated as nucleation sites and the finer is the final grain size. In a closed system (in absence of significant convective transport of solute, of grains and of inoculant particles) higher undercoolings are reached in alloys with a higher growth restriction factor and at higher cooling rates [3]. The grain size for a given alloy is in this case thus directly linked to the cooling rate. However, these tendencies are verified only in closed systems. But what happens when macroscopic convection phenomena appear? 
Only little research was made on the detailed modelling of nucleation in DC casting of aluminium alloys. Even when heterogeneous nucleation is considered in micro-macro models, grains are often considered to nucleate instantaneously at a unique temperature. We can name Håkonsen and coworkers [4], who tested different nucleation laws in their micro-macro model of DC casting and compared the model predictions with distributions of grain size measured experimentally for different $\mathrm{TiB}_{2}$ levels. Their model assumed that the grains move at the casting velocity, which corresponds to the assumption of a coalesced solid phase, without free-floating grains. Generally, we can say that the interactions between macroscopic transport phenomena and nucleation are not well known.

Thus in our work, using a fixed nucleation distribution, we propose to study the impact of combined transport phenomena on nucleation and growth of grains.

\section{Model description}

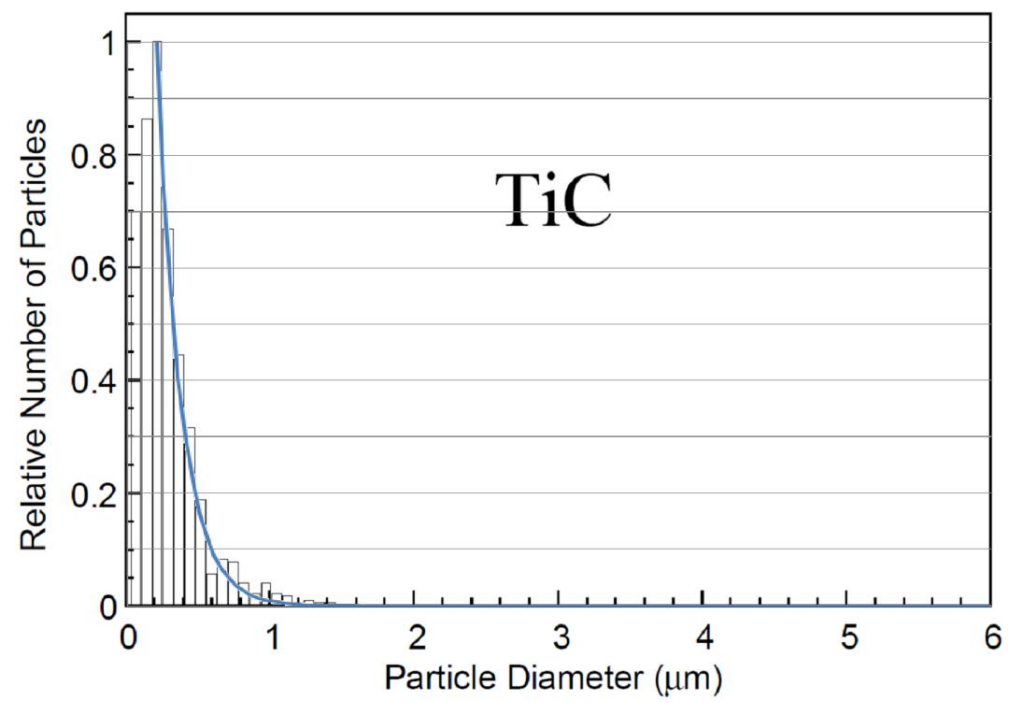

Figure 1. Size distribution of inoculant particles of $\mathrm{TiC}$ in the liquid metal for an Al-Ti-C grain refiner, given by Tronche [5], obtained experimentally (bar chart) and by numerical approximation (blue line).

To study the impact of inoculation on microstructure, we used the volume-averaged two-phase multiscale model SOLID. The model is described in detail in [6] and [7], so only its main features are presented here. Nucleation is supposed to be athermal, heterogeneous and instantaneous: grain refiners or inoculants, $\mathrm{TiB}_{2}$ or $\mathrm{TiC}$ particles, not soluble in the liquid alloy, act as potent nucleation sites. According to the athermal nucleation theory (or free growth) of Greer and coworkers [8], an inoculant particle of diameter $d$ is a site of nucleation if the undercooling reaches the critical activation undercooling $\Delta T_{c}(d)=\frac{4 \Gamma}{d} \propto d^{-1}$, where $\Gamma$ is the Gibbs-Thomson coefficient. In order to approximate the measured nuclei size distribution in the active range of inoculants, they used an exponential function $N(d)=\frac{N_{0}}{d_{0}} \exp \left(-\frac{d}{d_{0}}\right)$. According to [5], for $0.4 \mathrm{~kg} / \mathrm{t}$ of inoculant of type TiC, the distribution parameters are $N_{0}=5.31 \cdot 10^{13} \mathrm{~m}^{-3}$ and $d_{0}=1.66 \cdot 10^{-7} \mathrm{~m}$. This particle size distribution is shown in figure 1

In the model, this distribution is discretised into $n$ classes. For each class an inoculant density is associated to an activation undercooling, depending on the average inoculant size of the class. Moreover, the transport of nuclei is considered, assuming that the nuclei move at the velocity of the liquid. The conservation equation for nuclei of class $i$ is thus $\frac{\partial}{\partial t}\left(N_{n u c l}^{i}\right)+\nabla \cdot\left(\vec{v}_{l} N_{n u c l}^{i}\right)=\phi^{i}$, where $N_{n u c l}^{i}$ is the density of nuclei of class $i$ per unit volume, $\vec{v}_{l}$ is the intrinsic average liquid velocity and $\phi^{i}$ is the nucleation source term. The nucleation source term is a sink that removes the nuclei of class $i$ when 
the local undercooling becomes larger than the activation undercooling of class $i$; else, this term is null. The activated nuclei are transformed into grains. The conservation equation for spherical grains is $\frac{\partial N}{\partial t}+\nabla\left(\vec{v}_{s} N\right)=-\sum_{i} \phi^{i}$, where $N$ is the local grain density per unit volume and $\vec{v}_{s}$ is the velocity of the solid grains. The source term accounts for nucleation of grains from the inoculant particles.

The phase-change model describing the grain growth was slightly modified with respect to the original model of [6]. An improved expression for the liquid diffusion length, described in [9], was used. It provides a better description of the undercooling that results from the competition of nucleation and growth. An accurate description of the undercooling is essential for the proper evaluation of nucleation.

\section{Case definition and numerical details}

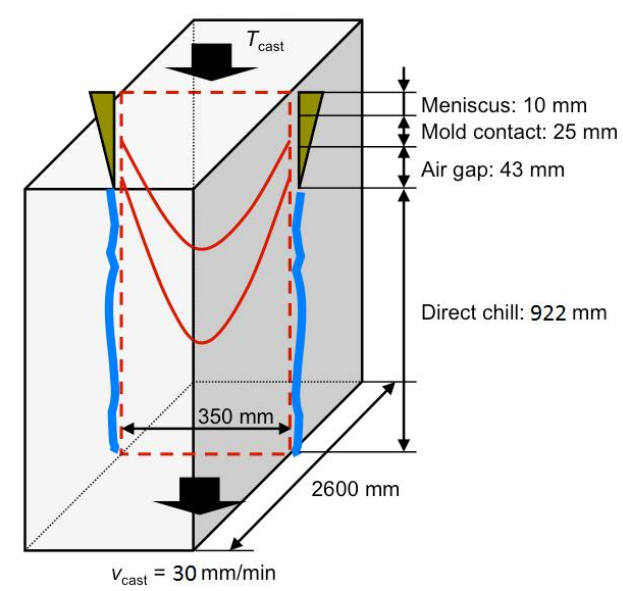

Figure 2. The 2D-ingot model geometry.

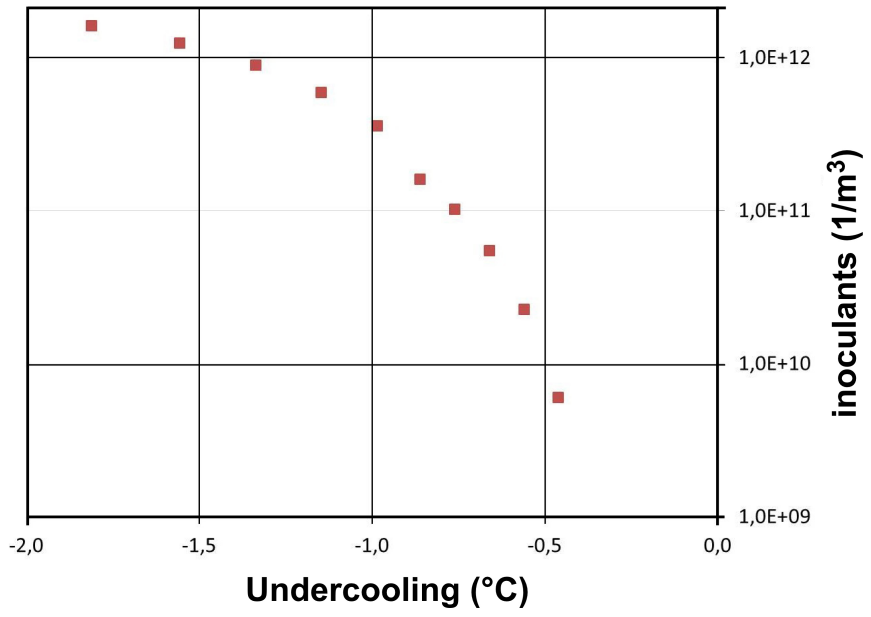

Figure 3. Discretisation of the inoculant distribution.

The solidification of a 7449 alloy ingot of $350 \mathrm{~mm}$ thickness was considered for this study. The geometry of the process is described in figure 2. The process parameters and the thermophysical properties are given in [7]. Only the casting speed and the mesh were changed. The speed was $30 \mathrm{~mm} / \mathrm{min}$ and the non uniform mesh was refined to $87 \times 120$. A $2 \mathrm{D}$ geometry with symmetry along the central axis was considered. To be able to assume that there is no heat extraction by the bottom, the ingot was studied along $1 \mathrm{~m}$. The dimensions of the computational domain are $X \times Y=175 \times 1000 \mathrm{~mm}$. In the liquid and mushy zones the cell size is $2 \times 2 \mathrm{~mm}$. The 7449 alloy, composed of more than 5 elements, was modeled in a simplified way by an equivalent binary alloy. The composition, the liquidus slope, the pure-Al melting temperature, the partition coefficient and solutal and thermal expansion coefficients were fitted in order to keep the same solidification path as for the multicomponent alloy. The inoculant distribution was discretised by 10 classes, as shown in figure 3 . This was done in such a way as to model the whole range of possible undercoolings. The first 5 classes were defined by a constant small undercooling step to capture them more precisely, then a geometrical ratio was used for larger undercoolings.

\section{Results and discussion}

In order to systematically investigate the impact of complicated and coupled convection phenomena on nucleation, we modeled the process with different simplifying hypotheses. In all cases we consider the coupling between the nucleation and the growth of the grains $(n g g)$. Then, we can account for the fluid flow induced by thermosolutal natural convection $(n c)$ and shrinkage $(s h)$ and for the motion of freefloating grains $(\mathrm{gm})$. We will present three cases in increasing order of complexity with respect to the physics of macroscopic transport: 


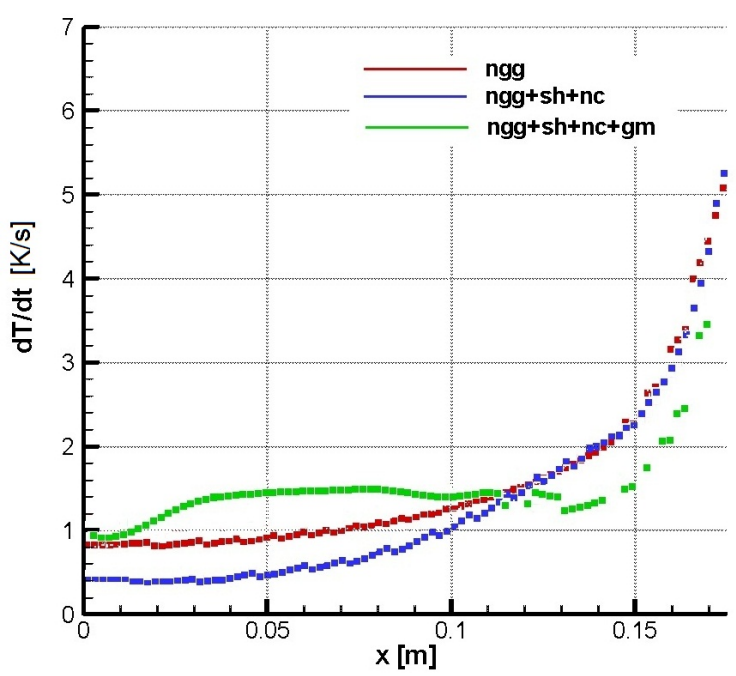

Figure 4. Profiles of cooling rate at the liquidus front obtained for the three physical hypotheses.

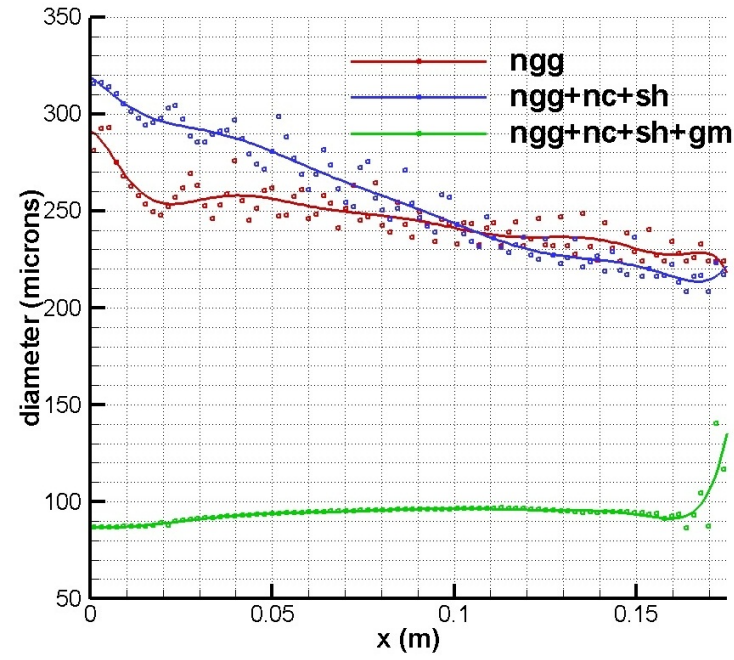

Figure 5. Profiles of average grain diameter obtained for the three physical hypotheses.

(i) we consider only the heat transfer, the nucleation and the growth of grains (ngg), and the motion of the product at the casting speed;

(ii) we take into account fluid flow with a fixed solid phase (ngg+nc+sh);

(iii) we additionally account for grain motion (ngg+nc+sh+gm).

For these three cases, profiles of cooling rate at the liquidus front (figure 4) and profiles of average grain diameter (figure 5) versus the distance from the centerline are provided.

Even if all relative motion between solid and liquid is neglected (case $n g g$ ), a not uniform grain size can be noticed throughout the product. Figure 5 shows that the grain diameter is larger at the center of the ingot and decreases quite regularly towards the surface. Figure 6 a shows the zone where the undercooling is high enough to activate the nuclei (the first class of nuclei is activated at $\Delta T_{c}=-0.4606^{\circ} \mathrm{C}$ ). Nucleation occurs in the whole thickness of the ingot, in a thin region next to the liquidus front. However, next to the surface higher undercoolings are reached and more nuclei classes are activated. By ignoring all the convective phenomena, the system of a growing grain can be considered as closed in the reference frame attached to the ingot, so the only cause of nonuniform undercooling is the cooling process. In DC casting the heat is extracted from the surface of the ingot; the cooling rate is thus faster closer to the surface. We can see this in Figure 4 , the cooling rate at the liquidus front along the ingot thickness is given. The obtained result is coherent with what was expected: the cooling rate being higher next to the surface, more nuclei classes are activated, so the average grain size is smaller.

Then, fluid flow due to natural convection and shrinkage is considered in case $n g g+n c+s h$. All growing grains are still moving at the casting speed, but the system of a growing grain cannot be considered as closed anymore. Other parameters than the cooling rate can alter the interactions between grain nucleation and growth. Figure 5 shows that the grain size is modified compared to case $n g g$, principally in the center of the ingot: it increases from $290 \mu \mathrm{m}$ to $310 \mu \mathrm{m}$. First of all, we can notice that the cooling rate is reduced in the center, as can be seen in figure 4. Due to the convective heat transfer by the natural convection flow (shown in figure $6 \mathrm{~b}$ ), the temperature in the center is more homogeneous, the thermal gradients are smaller and the cooling rate is thus lower. This thermal effect could explain the larger grain size in the center by a lower cooling rate. However, next to the surface, the cooling rate is the same if convection is taken into account or not, so other phenomena must be the reason why the grain size is decreased. We can observe and propose three possible mechanisms: 


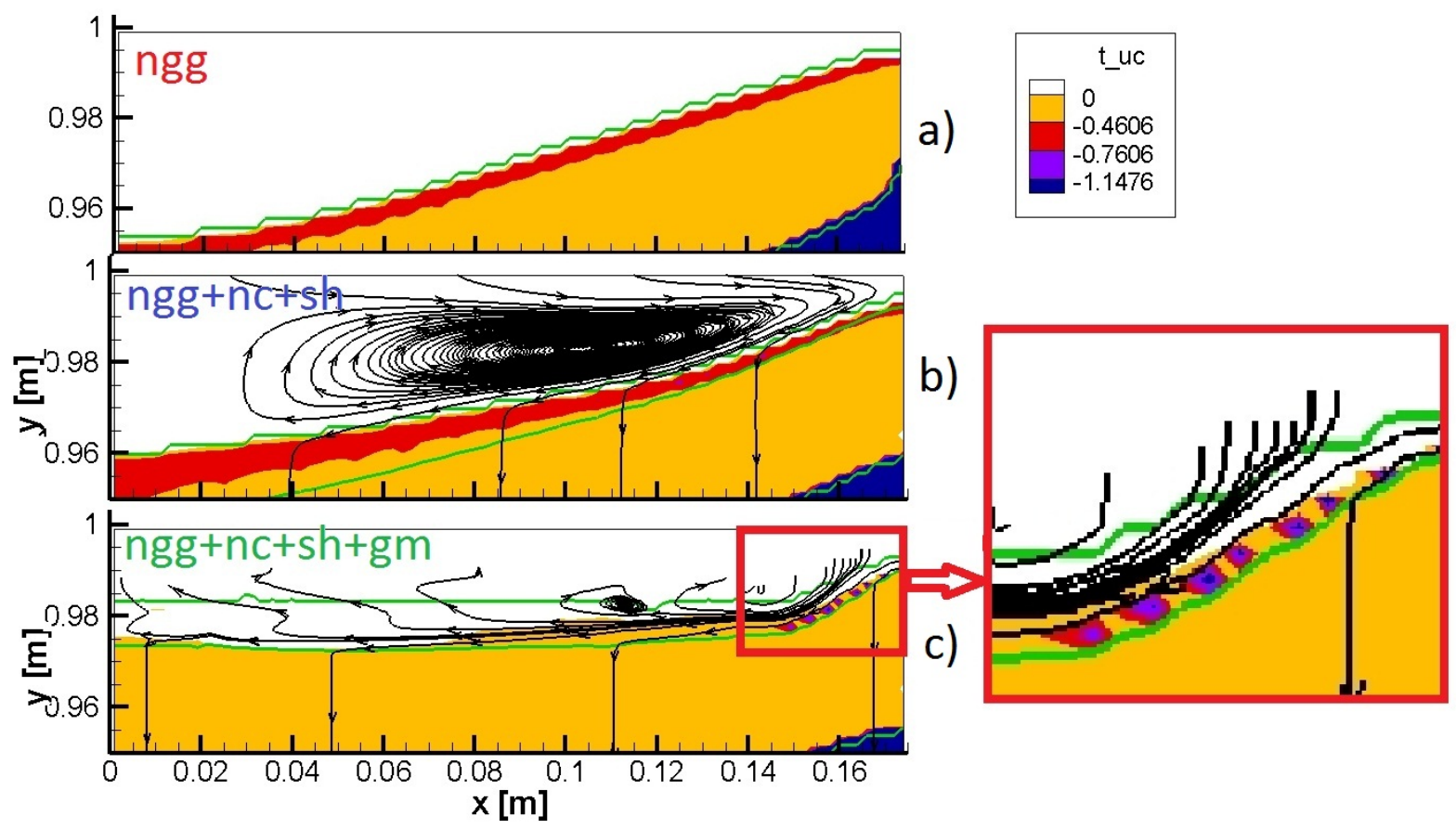

Figure 6. Undercooling map $\left({ }^{\circ} \mathrm{C}\right)$, isolines of solid fraction in green and streamlines in black. (a) ngg: solid fraction isolines 0 and 1, (b) ngg+sh+nc: solid fraction isolines 0 and 1 and liquid streamlines and (c) ngg+sh+nc+gm: solid fraction isolines $0,0.3$ and 1 and solid streamlines.

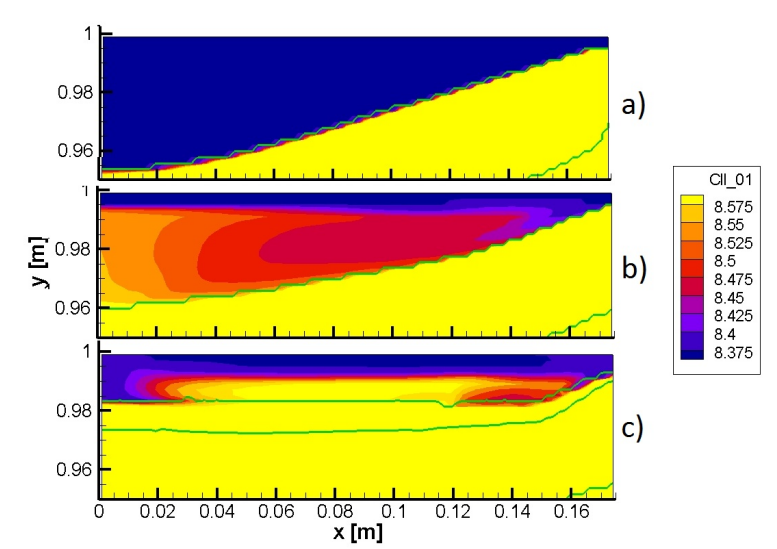

Figure 7. Average intrinsic liquid solute concentration (weight \%). (a) ngg, (b) ngg+sh+nc and (c) ngg+sh+nc+gm.

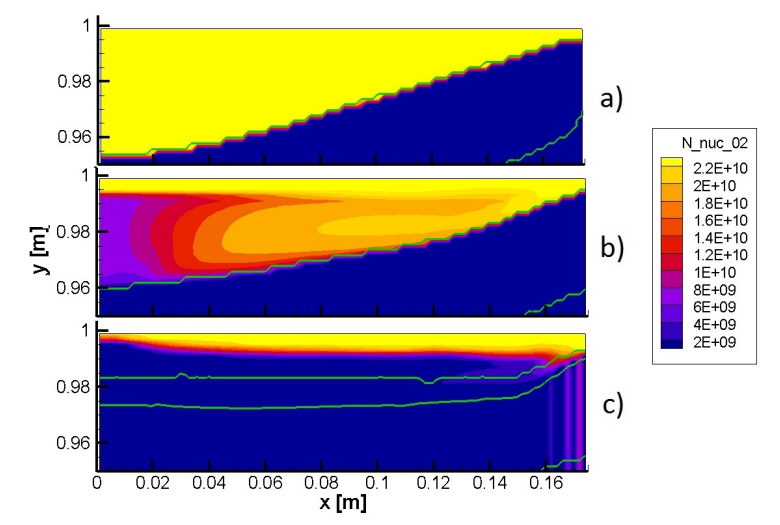

Figure 8. Nuclei density of the 2nd class (nuclei number $\left./ \mathrm{m}^{3}\right)$, activated when the undercooling reaches $-0.5606^{\circ} \mathrm{C}$ (nominal density:2.266 . $10^{10} \mathrm{~m}^{-3}$ ). (a) $\mathrm{ngg}$, (b) $\mathrm{ngg}+\mathrm{sh}+\mathrm{nc}$ and (c) ngg+sh+nc+gm.

(i) The liquid next to the liquidus front is enriched in solute due to solute rejection and fluid flow through the mushy zone, as shown in figure 7. Thus the growth restriction coefficient is larger, which increases the undercooling for a given cooling rate and could cause the nucleation of more grains. 
(ii) The flow circulation causes solute transport. In the zone close to the surface, solute lean liquid is flowing into the mushy zone to replace richer liquid and in the center zone solute-rich liquid from higher solid fractions is flowing towards the liquid sump to replace leaner liquid. This tends to increase or decrease the undercooling, respectively.

(iii) The nuclei density in the liquid pool is not homogeneous due to mixing of fresh melt from the inlet and melt that already went through the nucleation zone, where the nuclei are spent. Generally, the nuclei density in the center is smaller due to the direction of the flow circulation, as shown in figure 8 .

In case $n g g+n c+s h+g m$ grain motion is accounted for up to a solid packing fraction of 0.3 . We can see that the profile of grain size is largely modified (figure 5). The grains are much smaller and the size distribution is much more homogeneous. We can see that there is no clear relation between the grain size and the cooling rate any more (figures 4 and 5 ). This indicates that the grain transport seems to completely change the competition between nucleation and growth in the sump. Indeed, the grains are heavier than the liquid, so they settle, inducing a solid convection movement from the surface of the ingot to the center. In figure 6e we can see that undercoolings sufficient for nucleation are reached only in a small region located near the packing front and close to the surface. Grains that are formed there, continuously leave the nucleation region to settle in the center. This effectively reduces the local specific surface area of the grain interface (in a volume-averaged sense). A smaller surface of the solid-liquid interface decreases the efficiency of the solute diffusion from the grain interface into the surrounding liquid and therefore maintains a high solutal undercooling. We can observe undercoolings of around $-1.6^{\circ} \mathrm{C}$ against $-0.75^{\circ} \mathrm{C}$ in the cases without grain motion (figure 6c). The coupling of nucleation, growth and transport thus creates a zone highly favorable to nucleation, producing a large quantity of grains. On the opposite, in the ingot center, free-floating grains are continuously coming in from the nucleation region and accumulating. The high grain densities promote solute exchange at the grain interface and drastically lower the undercoolings. No nucleation occurs here. One can even notice a zone of remelting in the center of the ingot above the packing front.

In summary, we can observe that the nucleation zone is totally modified in the presence of grain motion: only a small zone, along the coherency line next to the surface, is the site of nucleation (figure 6). Moreover, the grain flow, illustrated in figure 6c shows that some grains settle or attach directly, while some grains first travel through the low-undercooling or remelting zone before packing. This could explain the duplex structure often observed in the center of ingots.

As a comment to the results we should note a hypothesis on grain remelting included in the model. We consider that inoculant particles from remelted grains remain in the melt and can act as potent nucleation sites (we attribute them to nuclei of the first class, with the lowest activation undercooling). This simplifying hypothesis leads to a strong increase (of about two orders of magnitude) of nuclei density of the first class in the totally liquid zone and is partly the reason for the strong reduction of the grain size. It does, however, not modify the fundamental idea of nucleation and growth zones, discussed above.

\section{Summary and conclusions}

We studied the impact of macroscopic convection phenomena on nucleation and grain structure formation for a 7449 alloy ingot of $350 \mathrm{~mm}$ thickness inoculated with $0.4 \mathrm{~kg} / \mathrm{t}$ of inoculant of type Al-Ti-C. We showed that even without any convection, the size of the grains is not uniform across the ingot. It follows the cooling rate: higher cooling rates close to the cooled surface lead to higher undercoolings and consequently to activation of more inoculant particles, which results in smaller grains. We show, however, that this classical explanation of the nucleation-growth competition (in terms of cooling rate) fails in the presence of convection phenomena. When liquid convection is considered, solute transport can alter the local undercooling. Furthermore, the transport of inoculant particles with the flow induces strong variations of local nuclei density in the nucleation zone. The grain motion has an even stronger 
impact and fundamentally changes the nucleation behavior as well as the grain size distribution in the ingot. The nucleation is limited to a small zone along the packing front next to the ingot surface and the large number of grains produced there is evenly distributed over the ingot thickness.

\section{References}

[1] Nadella R, Eskin D, Du Q and Katgerman L 2008 Progress in Materials Science 53421

[2] Nadella R, Eskin D and Katgerman L 2008 Metall. Mater. Trans. A 39A 450

[3] Dantzig J and Rappaz M 2009 Solidification (EPFL Press)

[4] Håkonsen A, Mortensen D, Benum S and Vatne H 1999 Light metals 1999 ed Eckert C E (Warrendale, USA: TMS) pp $821-827$

[5] Tronche A 2000 Investigation of Modelling of Inoculation of Aluminium by TiC Ph.D. thesis University of Cambridge

[6] Založnik M and Combeau H 2009 Comput. Mater. Sci. 481

[7] Založnik M, Kumar A, Combeau H, Bedel M, Jarry P and Waz E 2011 Adv. Eng. Mater. in press

[8] Greer A, Bunn A, Tronche A, Evans P and Bristow D 2000 Acta Mater. 482823

[9] Tveito K O, Bedel M, Založnik M, Combeau H and M'Hamdi M 2011 International Conference on Advances in Solidification Processes 3 (Aachen/Rolduc) 